\title{
ARTICLE
}

Clinical Study

\section{Validation of the IBIS breast cancer risk evaluator for women with lobular carcinoma in-situ}

\author{
Louisa Lisa Lo ${ }^{1}$, Roger Laughlin Milne ${ }^{2,3}$, Yuyan Liao ${ }^{4}$, Jack Cuzick ${ }^{5}$, Mary Beth Terry ${ }^{4,6}$ and Kelly-Anne Phillips (D) ${ }^{1,3,7,8}$
}

BACKGROUND: Management advice for women with lobular carcinoma in situ (LCIS) is hampered by the lack of accurate personalised risk estimates for subsequent invasive breast cancer (BC). Prospective validation of the only tool that estimates individual BC risk for a woman with LCIS, the International Breast Cancer Intervention Study Risk Evaluation Tool (IBIS-RET), is lacking.

METHODS: Using population-based cancer registry data for 732 women with LCIS, the calibration and discrimination accuracy of IBIS-RET Version 7.2 were assessed.

RESULTS: The mean observed 10-year risk of invasive BC was 14.1\% (95\% Cl:11.3\%-17.5\%). IBIS-RET overestimated invasive BC risk $(p=0.0003)$ and demonstrated poor discriminatory accuracy (AUC 0.54, 95\% Cl: $0.48-0.62$ ).

CONCLUSIONS: Clinicians should understand that IBIS-RET Version 7.2 may overestimate 10-year invasive BC risk for Australian women with LCIS. The newer IBIS-RET Version 8.0, released September 2017, includes mammographic density and may perform better, but validation is needed.

British Journal of Cancer (2018) 119:36-39; https://doi.org/10.1038/s41416-018-0120-z

\section{INTRODUCTION}

Women diagnosed with lobular carcinoma in-situ (LCIS) have an elevated risk of subsequent invasive breast cancer $(B C)^{1}$ that increases by about $1 \%$ every year after diagnosis to $13 \%$ after 10 years, $11 \%-26 \%$ at 15 years $^{1-3}$ and $21 \%-26 \%$ risk after 20 years. ${ }^{4,5}$

Most are managed with observation alone, ${ }^{6}$ but American Society of Clinical Oncology and Cancer Australia guidelines recommend that risk-reducing medications, specifically selective oestrogen receptor modulators (SERM) or aromatase inhibitors (Al) (the latter only in postmenopausal women), be discussed with LCIS patients. ${ }^{7-10}$ Risk-reducing bilateral mastectomy is pursued by only a minority of LCIS patients. ${ }^{11}$ Informed decision-making would be facilitated by accurate personalised risk estimates for future invasive BC.

The International Breast Cancer Intervention Study Risk Evaluation Tool (IBIS-RET) is the only tool available to estimate risk for an individual woman with LCIS. ${ }^{12-14}$ Although validated in other populations, ${ }^{15-17}$ IBIS-RET, to our knowledge, has not been validated in LCIS patients. Using population-based data, we prospectively examined the performance of IBIS-RET Version 7.2 for estimating invasive $B C$ risk for women with a history of LCIS.

\section{METHODS}

The Victorian Cancer Registry (VCR) has collected data on all cancer diagnoses in Victoria, Australia since 1982. It determines vital status of all registrants by record linkage to the state and national death registries. ${ }^{18}$ De-identified data including dates of birth, death, LCIS and invasive BC diagnoses were obtained from the VCR for all women diagnosed with pure LCIS between 1982 and 2015, when aged 20-70 years. 'Pure' LCIS was defined as LCIS without previous or synchronous ductal carcinoma in-situ (DCIS) or invasive BC in either breast (including within 6 months after LCIS diagnosis). Women with other invasive cancer diagnoses (except non-melanotic skin cancer) prior to their pure LCIS diagnosis were excluded. The study was approved by the Peter MacCallum Cancer Centre ethics committee.

The calibration and discriminatory accuracy of the 10-year IBISRET Version 7.2 estimates were assessed by comparing IBISRETassigned risks with observed invasive $B C$ incidence. To assess calibration, the mean IBIS-RET-assigned risk was compared with the mean 10-year observed invasive BC incidence in each IBIS-RETassigned risk group, using a chi-squared goodness-of-fit statistic, ${ }^{19}$ for the whole cohort (by tertiles) and also for two subgroups stratified by the diagnosis of LCIS before and at or after age 50 years. To evaluate discriminatory accuracy, the overall area under the receiver operating characteristic (ROC) curve for the development of invasive BC within 10 years of LCIS diagnosis was computed. RMAP (https://gailg.github.io/rmap/) and SAS software 9.4 (SAS Institute, Cary, NC) were used. Data were censored at date of invasive $B C$ diagnosis, death and date that the most recently linked death data were considered complete $\left(31^{\text {st }}\right.$ December 2015). Two exploratory analyses were also conducted; the first

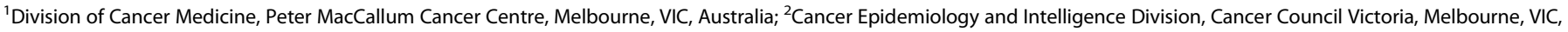

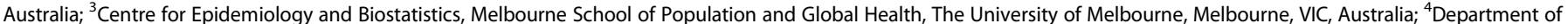

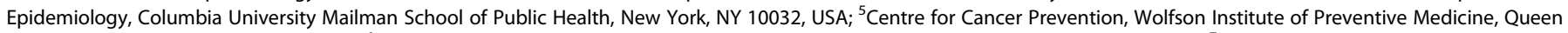

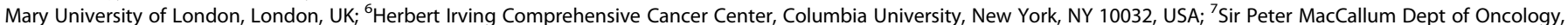
The University of Melbourne, Parkville, VIC 3053, Australia and ${ }^{8}$ Department of Medicine, St Vincent's Hospital, The University of Melbourne, Parkville, VIC 3053, Australia Correspondence: K-A. Phillips (Kelly.Phillips@petermac.org) 
censored the data at date of any ductal carcinoma in situ (DCIS) diagnosis and the second included diagnosis of either invasive breast cancer and/or DCIS as the primary endpoint.

\section{RESULTS}

There were 732 eligible women (median age at LCIS 50 years, range 25-70 years, mean follow-up 9.8 years, range 0.04-33.9 years, total 4855 person-years), of whom 73 were diagnosed with invasive BC within 10 years after their LCIS. 10 women died within 10 years without an invasive BC diagnosis, 293 women were invasive $B C$-free at 10 years and 356 women were last observed without invasive $\mathrm{BC}$ with less than 10 years follow-up. The mean observed risk of invasive BC at 10 years was $14.1 \% \quad(95 \%$ confidence interval (Cl) $11.3 \%-17.5 \%$ ), whilst the mean assigned IBIS-RET 10-year risk was $20.9 \%$.

Figure 1 shows the cumulative invasive $B C$ incidence by 10 -year IBIS-RET-assigned risk tertile (i.e. $<18.8 \%, \geq 18.8 \%-<23.5 \%$, $\geq 23.5 \%$ ).

Figure 2 shows that the mean IBIS-RET-assigned invasive BC risks by tertile were significantly different to the observed $B C$ incidence at 10 years $(p=0.0003)$. Overall the IBIS-RET Version 7.2 tended to overestimate invasive $B C$ risk. When we compared the calibration for women below and above age 50 using internal

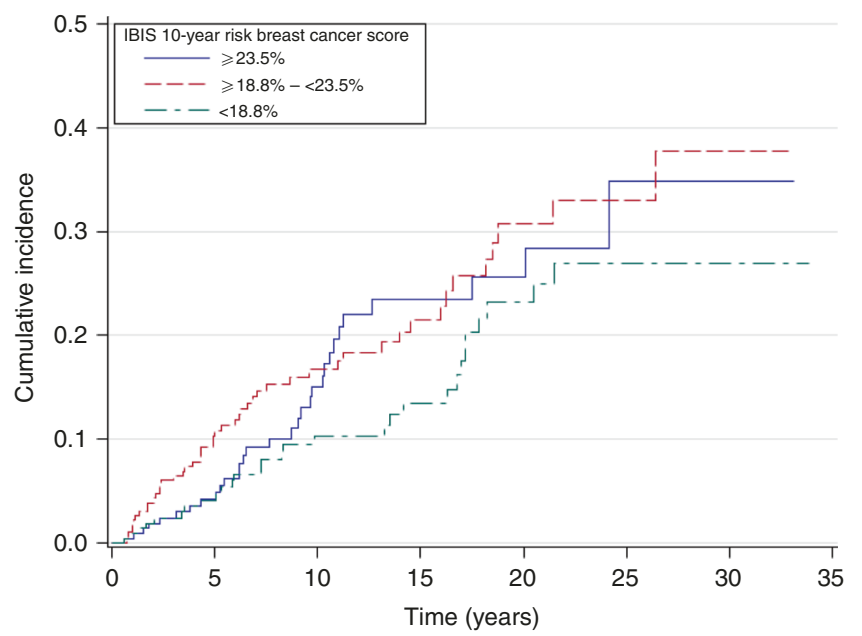

Fig. 1 Cumulative incidence of invasive breast cancer in patients with LCIS, by tertile of IBIS-RET estimated risk cutpoints by age, we found it was well-calibrated for women diagnosed before age 50 years $(p=0.13)$, but not for those diagnosed at or after age 50 years mainly owing to the lack of fit and overestimation by IBIS-RET for older women in the highest quantile, $(p=0.00007)$ (Fig. 3a,b).

The area under the ROC curve (AUC) for the IBIS-RET 10-year invasive $\mathrm{BC}$ risk estimates was 0.54 ( $95 \% \mathrm{Cl}: 0.48-0.62)$ overall, and 0.55 (95\% Cl: $0.44-0.0 .63)$ and 0.48 (95\% Cl: $0.38-0.57)$ for women diagnosed with LCIS before age 50 years and at or after age 50 years, respectively.

Nine women were diagnosed with DCIS during the first 10 years after their LCIS diagnosis. The results were similar when the analyses were repeated either censoring at DCIS diagnosis or including the 9 cases of DCIS along with the invasive BC cases in the primary endpoint (Supplementary Figure 1).

\section{DISCUSSION}

In this population-based study of women with LCIS, the IBIS-RET Version 7.2 tended to overestimate 10-year invasive BC risk and had poor discriminatory accuracy. However this study had several limitations that may have contributed to this finding.

The lack of information in our dataset regarding uptake of bilateral mastectomy or risk-reducing medication after LCIS diagnosis could have resulted in our study erroneously finding that IBIS-RET overestimates BC risk. However, uptake of these interventions is historically very low in Australia, even in very high-risk women, ${ }^{20}$ so this is unlikely to have been a major factor.

Histopathological diagnostic thresholds for atypical hyperplasia (AH) and LCIS have changed over time. ${ }^{21}$ If some of the cases included in this study were in fact $\mathrm{AH}$ misclassified as LCIS, this could have contributed to our finding that IBIS-RET V7.2 overestimates $B C$ risk, because $A H$ confers a lower $B C$ risk than LCIS. No pathology review of cases was undertaken by the authors or the VCR.

The IBIS-RET model is calibrated to UK BC incidence rates for 2008-2010 (Supplementary Table 1). ${ }^{22}$ Although our study covers Victorian women over a period from 1982-2015, the average agespecific BC incidence figures for these Victorian women closely resembled those used in the IBIS-RET model, except for the lower incidence in those aged 50 years and over (Supplementary Table 1). ${ }^{23}$ This could have contributed to the overestimation and poorer calibration in our dataset for women diagnosed with LCIS at and over age 50 years, but it is unlikely to completely explain our findings.

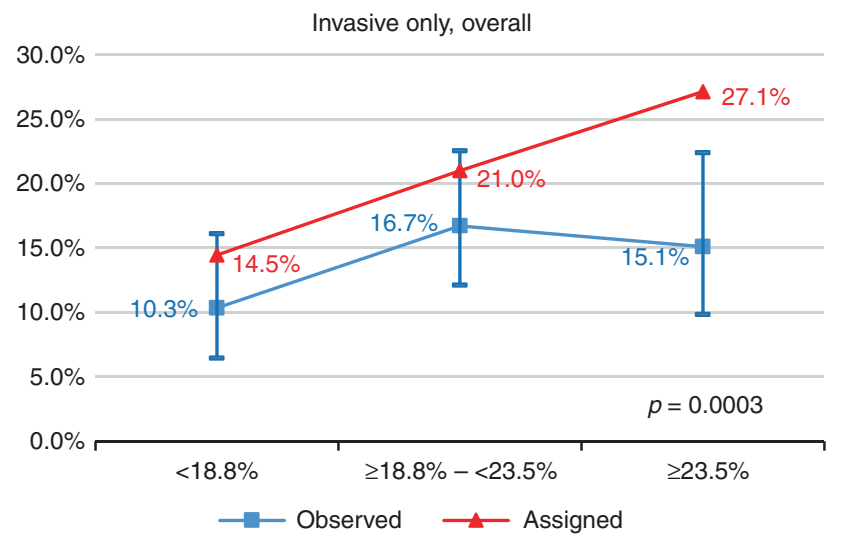

Fig. 2 Calibration of IBIS-RET for estimates of invasive BC in women with LCIS. The assigned line (triangle symbol) is the mean 10-year predicted risks of the IBIS-RET for that tertile $(<18.8 \%, \geq 18.8 \%-<23.5 \%, \geq 23.5 \%)$. The observed line (square symbol) is the estimates of 10 -year breast cancer probabilities based on the womens' observed breast cancer status, and the bars denote $95 \%$ confidence intervals for the observed risk 
a

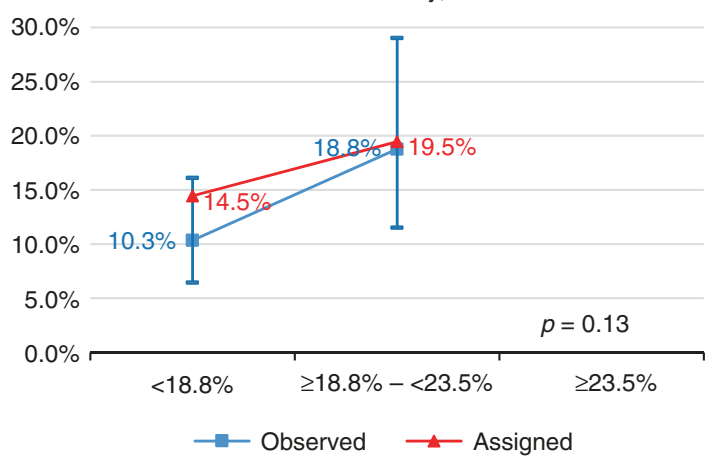

b

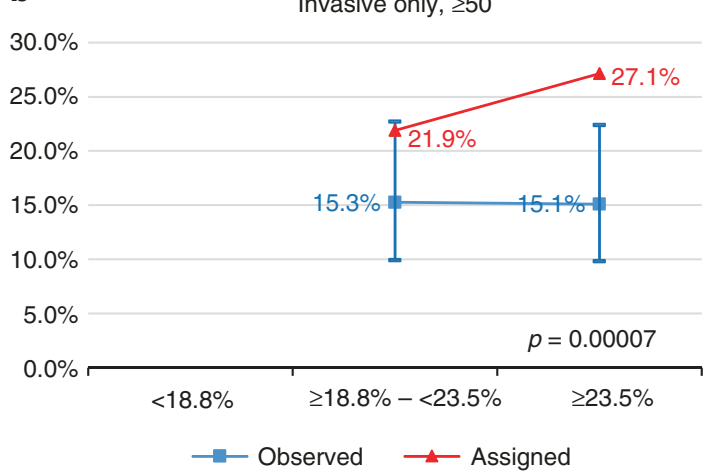

Fig. 3 a Calibration of IBIS-RET for estimates of invasive BC in women with LCIS diagnosed at $<50$ years (cut-off: $<18.8 \%, \geq 18.8 \%-<23.5 \%$ ). b Calibration of IBIS-RET for estimates of invasive BC in women with LCIS diagnosed at $>=50$ years (cut-off: $\geq 18.8 \%-<23.5 \%, \geq 23.5 \%$ ). The assigned line (triangle symbol) is the mean 10-year predicted risks of the IBIS-RET for that tertile $(<18.8 \%, \geq 18.8 \%-<23.5 \%, \geq 23.5 \%)$. The observed line (square symbol) is the estimates of 10-year breast cancer probabilities based on the womens' observed breast cancer status, and the bars denote $95 \%$ confidence intervals for the observed risk

Overdiagnoses from mammographic screening could have influenced our findings. However, Victorian women aged 50 years and over are screened 2-yearly as opposed to 3-yearly in the UK and this higher screening frequency should, if anything have resulted in a higher invasive $B C$ incidence when compared to the UK population, but this was not observed in those aged older than 50 years (Supplementary Table 1 ).

Patient migration out of Victoria after LCIS diagnosis would mean that some subsequent invasive breast cancers were not captured in the VCR data. Using aggregate data from the Australian Institute of Health and Welfare, we estimated that this interstate migration would have resulted in approximately 3 cases of invasive cancer being missed in our dataset, which could in part have contributed to our finding that IBIS-RET overestimates BC risk.

The study dataset also did not include information on other BC risk factors, but because IBIS-RET Version 7.2 relies only on age to estimate subsequent $B C$ risk in women with LCIS, this would not have impacted our findings. In fact there are conflicting reports on whether age at LCIS diagnosis affects subsequent BC risk. One study reported that the relative risk of $B C$ tended to decrease with increasing age at LCIS diagnosis. ${ }^{4}$ Conversely, according to King et al, ${ }^{3}$ risk factors like family history, age and breast density were not associated with BC risk in women with LCIS. Instead, the authors found that chemoprevention was the major factor associated with lower $\mathrm{BC}$ risk (Hazard ratio, $0.27 ; 95 \% \mathrm{Cl}, 0.15$ to 0.50). They also performed a subgroup nested case-control analysis, which showed that the volume of LCIS, which was defined as the ratio of slides with LCIS to total number of slides reviewed, was associated with $B C$ development $(p=0.008)$. Therefore, volume of LCIS might provide further risk stratification in women with LCIS.

Mammographic density is an important risk factor for breast cancer and has been shown to refine the IBIS-RET model in predicting $B C$ risk for women at increased risk, although not specifically in women with LCIS. ${ }^{24} \mathrm{~A}$ subsequent study using a UK prospective BC screening cohort showed that using mammographic density with the IBIS-RET improved the accuracy of BC risk prediction. ${ }^{25}$

Since starting this study, IBIS-RET, Version $8^{22}$ has been released (September 2017). For women with LCIS, Version 8 differs from Version 7.2 in that it now uses cancer family history and mammographic density (if available) to predict BC risk, as well as age at diagnosis of LCIS. The addition of cancer family history means that, in the absence of mammographic density information, IBIS-RET Version 8 will always provide the same or higher 10year BC risk estimates as Version 7.2 (which we have shown here already tends to overestimate risk). However, if mammographic density is known to be low, the risk estimate provided by Version 8 may be lower than that provided by Version 7.2. ${ }^{25} \mathrm{~A}$ validation study of IBIS-RET Version 8, using a dataset of women with LCIS and known mammographic density and family history, is highly desirable.

\section{ACKNOWLEDGEMENTS}

We thank Helen Farrugia for her assistance in acquiring study data and Adam Brentnall for his valuable insights during the data interpretation phase of this study.

\section{AUTHOR CONTRIBUTIONS}

Conception and design - LLL, KAP. Acquisition of data - RLM. Data analysis - YL. Interpretation of data-LLL, RLM, YL, JC, MBT, KAP. Drafting and revising manuscript - LLL, RLM, YL, MBT, KAP. Final approval of manuscript-LLL, RLM, YL, JC, MBT, KAP.

\section{ADDITIONAL INFORMATION}

Supplementary information is available for this paper at https://doi.org/10.1038/ s41416-018-0120-z.

Competing interests: The IBIS model is offered for commercial use by Cancer Research UK and Dr Cuzick receives a portion of the derived royalties. All other authors of this paper have declared no conflict of interest.

Funding: KAP is an Australian National Breast Cancer Foundation Practitioner Fellow.

Availability of data and material: The data used for this study are available from the Victorian Cancer Registry but restrictions apply to the availability of these data, which were used for the current study with appropriate approvals, and so are not generally publicly available. Data are however available from the authors upon reasonable request and with permission of the Victorian Cancer Registry.

\section{REFERENCES}

1. Page, D. L., Kidd, T. E. Jr, Dupont, W. D., Simpson, J. F. \& Rogers, L. W. Lobular neoplasia of the breast: higher risk for subsequent invasive cancer predicted by more extensive disease. Hum. Pathol. 22(12), 1232-1239 (1991).

2. Chuba, P. J. et al. Bilateral risk for subsequent breast cancer after lobular carcinoma-in-situ: analysis of surveillance, epidemiology, and end results data. J. Clin. Oncol. 23(24), 5534-5541 (2005).

3. King, T. A. et al. Lobular carcinoma in situ: A 29-year longitudinal experience evaluating clinicopathologic features and breast cancer risk. J. Clin. Oncol. 33(33), 3945-3952 (2015). 
4. Bodian, C. A., Perzin, K. H. \& Lattes, R. Lobular neoplasia. Long term risk of breast cancer and relation to other factors. Cancer 78(5), 1024-1034 (1996).

5. To, T., Wall, C., Baines, C. J. \& Miller, A. B. Is carcinoma in situ a precursor lesion of invasive breast cancer. Int. J. Cancer. 135(7), 1646-1652 (2014).

6. Degnim, A. C. \& King, T. A. Surgical management of high-risk breast lesions. Surg. Clin. North. Am. 93(2), 329-340 (2013).

7. Fisher, B. et al. Tamoxifen for prevention of breast cancer: report of the National Surgical Adjuvant Breast and Bowel Project P-1 Study. J. Natl. Cancer. Inst. 90(18), 1371-1388 (1998).

8. Vogel, V. G. et al. Effects of tamoxifen vs raloxifene on the risk of developing invasive breast cancer and other disease outcomes: the NSABP Study of Tamoxifen and Raloxifene (STAR) P-2 trial. JAMA. 295(23), 2727-2741 (2006).

9. Visvanathan, K. et al. Use of pharmacologic interventions for breast cancer risk reduction: American Society of Clinical Oncology clinical practice guidelines. J. Clin. Oncol. 31(23), 2942-2962 (2013).

10. Cancer Australia. Clinical guidance for the management of lobular carcinoma in situ [Internet]. (Cancer Australia, NSW (AU), 2016. https://canceraustralia.gov.au/ publications-and-resources/clinical-practice-guidelines/clinical-guidancemanagement-lobular-carcinoma-situ [cited 2017 Nov 10]. Available from.

11. Oppong, B. A. \& King, T. A. Recommendations for women with lobular carcinoma in situ (LCIS). Oncology (Williston Park) 25, (1051-1056 (2011). 1058.

12. Tyrer, J., Duffy, S. W. \& Cuzick, J. A breast cancer prediction model incorporating familial and personal risk factors. Stat. Med. 23(7), 1111-1130 (2004).

13. Amir, E., Freedman, O. C., Seruga, B. \& Evans, D. G. Assessing women at high risk of breast cancer: A review of risk assessment models. J. Natl. Cancer. Inst. 102(10), 680-691 (2010).

14. U.S. Preventive Services Task Force. Final Recommendation Statement: Breast Cancer: Medications for Risk Reduction [Internet]. (U.S. Preventive Services Task Force, Rockville (US), 2016. https://www.uspreventiveservicestaskforce.org/Page/ Document/RecommendationStatementFinal/breast-cancer-medications-for-riskreduction [cited 2017 Nov 10]. Available from.

15. Amir, E. et al. Evaluation of breast cancer risk assessment packages in the family history evaluation and screening programme. J. Med. Genet. 40(11), 807-814 (2003).

16. Jacobi, C. E., de Bock, G. H., Siegerink, B. \& van Asperen, C. J. Differences and similarities in breast cancer risk assessment models in clinical practice: which model to choose? Breast. Cancer. Res. Treat. 115(2), 381-390 (2009).

17. Quante, A. S., Whittemore, A. S., Shriver, T., Strauch, K. \& Terry, M. B. Breast cancer risk assessment across the risk continuum: genetic and nongenetic risk factors contributing to differential model performance. Breast. Cancer. Res. 14(6), R144 (2012).
18. Cancer Council Victoria. Victorian Cancer Registry [Internet]. (Cancer Council Victoria, Melbourne (AU), 2017. http://www.cancervic.org.au/research/registrystatistics/vcr [cited 2017 Nov 11]. Available from.

19. Whittemore, A. S. \& Halpern, J. Two-stage sampling designs for external validation of personal risk models. Stat. Methods. Med. Res. 25(4), 1313-1329 (2016).

20. Collins, I. M. et al. Preventing breast and ovarian cancers in high-risk BRCA1 and BRCA2 mutation carriers. Med. J. Aust. 199(10), 680-683 (2013).

21. Ginter, P. S. \& D'Alfonso, T. M. Current concepts in diagnosis, molecular features, and management of lobular carcinoma in situ of the breast with a discussion of morphologic variants. Arch. Pathol. Lab. Med. 141(12), 1668-1678 (2017).

22. Cuzick, J. IBIS Breast Cancer Risk Evaluation Tool [Internet]. (Cuzick, J, London (UK), 2017. http://www.ems-trials.org/riskevaluator/ [cited 2017 Nov 8]. Available from.

23. Cancer Council Victoria. Victorian Cancer Statistics. Age-specific cancer rates, Victoria (Incidence)[Internet]. (Cancer Council Victoria, Melbourne (AU), 2017. http:// vcrdata.cancervic.org.au/vs/ [cited 2017 Nov 8]. Available from.

24. Warwick, J. et al. Mammographic breast density refines Tyrer-Cuzick estimates of breast cancer risk in high-risk women: findings from the placebo arm of the International Breast Cancer Intervention Study I. Breast. Cancer. Res. 16(5), 451 (2014).

25. Brentnall, A. R. et al. Mammographic density adds accuracy to both the TyrerCuzick and Gail breast cancer risk models in a prospective UK screening cohort. Breast. Cancer. Res. 17(1), 147 (2015).

(i) Open Access This article is licensed under a Creative Commons Attribution 4.0 International License, which permits use, sharing adaptation, distribution and reproduction in any medium or format, as long as you give appropriate credit to the original author(s) and the source, provide a link to the Creative Commons license, and indicate if changes were made. The images or other third party material in this article are included in the article's Creative Commons license, unless indicated otherwise in a credit line to the material. If material is not included in the article's Creative Commons license and your intended use is not permitted by statutory regulation or exceeds the permitted use, you will need to obtain permission directly from the copyright holder. To view a copy of this license, visit http://creativecommons. org/licenses/by/4.0/.

(c) The Author(s) 2018 